\title{
CARACTERIZAÇÃO DAS PROPRIEDADES FÍSICO-MECÂNICAS DA MADEIRA DE EUCALIPTO COM DIFERENTES DENSIDADES ${ }^{1}$
}

\author{
Moisés Silveira Lobão², Ricardo Marius Della Lúcia², Márcio Sampaio Sarmet Moreira e Adriana Gomes²
}

\begin{abstract}
RESUMO - O presente trabalho teve por objetivo caracterizar um lote de madeira de eucalipto comercializada por uma empresa do setor florestal brasileiro. Utilizou-se, neste trabalho, a madeira de Eucalyptus, provavelmente E. grandis W. Hill ex Maiden., adquirida no mercado local e no estado de secada ao ar. Todo o lote foi dividido em dois sublotes, um de madeira mais pesada e outro da mais leve, porque se percebeu, no recebimento do carregamento, que havia nele tábuas com diferenças notáveis de peso. Os corpos-de-prova foram preparados a partir de tábuas retiradas ao acaso de cada um desses dois sublotes. Fez-se, portanto, a caracterização dessa madeira para possíveis comparações dos valores de suas propriedades físicas e mecânicas, segundo a norma NBR 7190 (1997). Feitos os testes, de fato pôde-se verificar a existência de duas populações de tábuas com propriedades mecânicas significativamente diferentes.
\end{abstract}

Palavras-chave: Madeira comercial, densidade e NBR 7190.

\section{CHARACTERIZATION OF PHYSICAL AND MECHANICAL PROPERTIES OF EUCALYPTUS LUMBER WITH DIFFERENT SPECIFIC GRAVITIES}

\begin{abstract}
The objective of the present work was to characterize a lot of Eucalyptus wood marketed by a Brazilian forest company. In this work, Eucalyptus wood, probably E. grandis W. Hill ex Maiden, was purchased in the local market in the air-dry condition. The whole lot was subdivided in two sub-lots; one with heavier wood, the other with lighter. The reason for this was that the shipment arrived with boards having notable differences in weight. Test-specimens were prepared from boards taken from each sub-lot. The characterization was therefore carried out in twice as many test-specimens as required by Brazilian standards, as described in the NBR 7190 (1997) standard. The tests showed the existence of two board populations with significantly different mechanical properties.
\end{abstract}

Key words: Lumber, specific gravity and NBR 7190

\section{INTRODUÇÃO}

Vários estudos têm procurado identificar quais os fatores que afetam as propriedades físico-mecânicas da madeira e os que podem ser inerentes à própria madeira, como também ao ambiente onde a árvore se desenvolve.

Sabe-se que as propriedades mecânicas da madeira são dependentes, principalmente, da densidade básica, da porcentagem de madeira juvenil, da largura dos anéis, do ângulo das microfibrilas, da inclinação da grã, da quantidade de extrativos, do teor de umidade, da intensidade ao ataque de insetos, do tipo e da localização e quantidade de nós, dentre outros fatores (EVANS et al., 2000).

Comojá se sabe, a madeira é um material heterogêneo, possuindo diferentes tipos de células, adaptadas ao desempenho de funções específicas; as variações nas suas composições químicas, físicas e anatômicas são

\footnotetext{
${ }^{1}$ Recebido em $1^{\circ} .09 .2003$ e aceito para publicação em 10.08.2004.

${ }^{2}$ Departamento de Engenharia Florestal da UFV, 36570-000 Viçosa, MG. E-mail: <rdlucia@ufv.br>.

${ }^{3}$ Departamento de Engenharia Civil da UFV, 36570-000. Viçosa, MG.
} 
grandes entre espécies. Dentro da mesma espécie, elas também ocorrem conforme a idade, posição na árvore, fatores genéticos e ambientais etc. (SHIMOYAMA, 1990). Esse mesmo autor afirmou que o ambiente, bem como sua manipulação, pode afetar as características da madeira e, conseqüentemente, sua qualidade. As propriedades físico-mecânicas da madeira de árvores plantadas em um ambiente exótico podem ser significativamente diferentes, muitas vezes, daquelas cultivadas em um ambiente nativo.

Dados de trabalhos anteriores Panshin e De Zeeuw (1980), Evans et al. (2000) e Tomazello Filho (1985a) indicaram que existem importantes variações nas propriedades físico-mecânicas entre as árvores, mesmo quando são originadas do mesmo clone e do mesmo sítio. Isso se deve à característica biológica das árvores, a qual possui fontes naturais de variação que, muitas vezes, não podem ser eliminadas.

Segundo a Eucalipto (2003), as principais propriedades físicas e mecânicas da madeira que provocam impacto na qualidade do produto final a ser produzido são identificadas como: a) resistência mecânica, b) massa específica aparente e c) estabilidade dimensional, e os principais impactos tecnológicos nas características das madeiras passam pelo melhoramento florestal e práticas silviculturais e de manejo, além das etapas de processamentos primário e secundário. Nessa mesma fonte, comentou-se que existe unanimidade entre os pesquisadores da área de produtos florestais de que a qualidade da madeira para determinados usos pode ser melhorada, modificada ou ter alguns fatores minimizados ou controlados, em considerável extensão, se houver um trabalho conjunto dos setores de produção florestal e industrial, integrando os silvicultores com os engenheiros e os técnicos da área de processamento.

Segundo Shioyama (1990), a densidade é uma quantificação direta do material lenhoso por unidade de volume, estando relacionada a muitas propriedades e características tecnológicas fundamentais para a produção e utilização dos produtos florestais, um dos parâmetros mais importantes entre as diversas propriedades físicas da madeira, pois afeta todas as demais propriedades desta. Seus efeitos, porém, são interativos e difíceis de serem avaliados isoladamente. Segundo Panshin e De Zeeuw (1980), há uma diminuição da densidade no sentido base-topo, à exceção das espécies de bases expandidas, que crescem em regiões alagadas e, conseqüentemente, apresentam aumento da densidade no sentido casca-medula.

Tomazello Filho (1985) verificou, em amostras de madeira de Eucalyptus grandis W. Hill ex Maiden., que a região central do tronco apresentava densidades mais baixas próximo da medula e menores variações ao longo do raio, com tendência à formação de anéis de crescimento mais largos próximo à periferia e, conseqüentemente, ocorriam grandes alterações na densidade na direção radial dessa árvore.

Latorraca et al. (2000) observaram que as propriedades da madeira variam conforme o crescimento da árvore, com a ressalva de que a porção da madeira correspondente aos primeiros anéis formados apresenta menor massa específica e fibras mais curtas, entre outras características, ou seja, a diferença entre lenho juvenil e adulto. Verificaram que há aumento da massa específica, bem como da espessura da parede celular em anéis sucessivos, a partir do centro para a periferia do tronco da árvore, no sentido medula-casca. Também foi verificado que a densidade tende a aumentar com a idade, com consequiente aumento da espessura da parede celular e diminuição da largura das células.

Segundo Panshin e De Zeeuw (1980), a densidade básica em função da idade da árvore em geral aumenta rapidamente durante o período juvenil, depois mais lentamente até atingir a maturidade, quando permanece mais ou menos constante, e a madeira de lenho tardio apresentava densidade básica maior que a de lenho juvenil. Esses mesmos autores afirmaram que a variabilidade da maior parte das propriedades mecânicas da madeira pode ser estimada com base na variação da densidade. Isso pode ser explicado porque as propriedades de resistência à flexão estática e o seu MOE (módulo de elasticidade) estão correlacionados com a densidade, a qual, por sua vez, também está correlacionada com as dimensões das células. Mencionaram também que a relação entre densidade e as propriedades mecânicas pode ser alterada pela presença de extrativos que se adicionam à massa lenhosa, aumentando, principalmente, a resistência à compressão axial da madeira, e a efetiva resistência desta a alguma forma particular de aplicação de esforços é uma função não só da quantidade total da parede celular, mas também da proporção dos componentes das células estabelecidas em dada espécie e da quantidade de extrativos presentes no lúmen das células.

Segundo Norman (1972), a espessura definitiva da parede das fibras ocasiona somente uma dureza

\section{R. Árvore, Viçosa-MG, v.28, n.6, p.889-894, 2004}

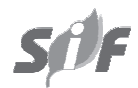


mais elevada de uma madeira, mas sem afetar, de maneira marcante, a sua densidade e suas propriedades mecânicas. Segundo Meyer (1930), citado por Gonçalez (1993), a proporção de raios tem ligação com a dureza e com a resistência em compressão axial e transversal da madeira.

Quando se pensa em espécies de rápido crescimento como alternativa para produção de madeira, o gênero Eucalyptus se apresenta como uma opção potencial das mais importantes não somente por sua capacidade produtiva e adaptabilidade a diversos ambientes, mas, sobretudo, pela grande diversidade de espécies, tornando possível atender aos requisitos tecnológicos dos mais diversos segmentos da produção industrial madeireira. São várias as razões para que o eucalipto possa ser indicado como alternativa de oferta de madeira. O gênero tem ocupado um lugar preferencial na escolha de espécies para o estabelecimento de florestas plantadas no país. Na região centro-sul brasileira nas últimas décadas, observou-se um vasto e bem-sucedido programa de reflorestamento com essas espécies. Apesar de a maior parte das florestas estar comprometida com a produção de madeira para os denominados "usos tradicionais" (celulose, papel, carvão vegetal, lenha e chapas de fibras), espera-se que uma parcela possa ser destinada a outras aplicações madeireiras. Para atender a tais demandas, uma primeira etapa envolveu uma seleção de espécies com programas de melhoramento para atender a características de ordem silvicultural, como crescimento, forma do tronco, regeneração e resistência a pragas e doenças; numa segunda etapa, tais programas foram consolidados e complementados com o envolvimento de algumas propriedades da madeira, como densidade, dimensões de fibras, teores de casca e composição química (EUCALIPTO, 2001).

Segundo a Eucalipto (2003), o Brasil consome atualmente 350 milhões de $\mathrm{m}^{3} /$ ano, o que significa $8 \%$ do consumo mundial de madeira. Estudos mais recentes têm indicado que existem no país 4,6 milhões de ha de florestas plantadas, sendo 1,7 milhão do gênero Pinus e 2,9 milhões do gênero Eucalyptus. Segundo os autores citados anteriormente, no Brasil as espécies de eucalipto mais utilizadas são Eucalyptus grandis (55\%), Eucalyptus saligna Sm. (17\%), Eucalyptus urophylla S.T. Blake (9\%), Eucalyptus viminalis Hook. (2\%), híbridos de Eucalyptus grandis $x$ E. urophylla (11\%) e outras espécies (6\%). O potencial de utilização múltipla da madeira de eucalipto tem crescido, sobremaneira, nos últimos tempos, principalmente se adotar o conceito de floresta de aplicação ampla, ou seja, podem-se conseguir diferentes tipos de floresta para cada um dos produtos a serem obtidos, havendo a possibilidade de se explorar todo o potencial do gênero Eucalyptus.

Segundo a Eucalipto (2001), entre as espécies de eucalipto comercialmente utilizadas, o Eucalyptus grandis é a que apresenta o melhor comportamento físico-mecânico para uso da madeira sólida.

Nesse contexto, objetivou-se com este trabalho realizar uma caracterização físico-mecânica da madeira de eucalipto, provavelmente $E$. grandis, obtida no mercado local, utilizando a norma NBR7190/97, para verificar as possíveis influências da densidade sobre as propriedades mecânicas dessa madeira.

\section{MATERIAL E MÉTODOS}

O trabalho foi desenvolvido no Laboratório de Propriedades Físico-Mecânicas da Madeira do Departamento de Engenharia Florestal da UFV, que dispunha do equipamento necessário para caracterização das propriedades físico-mecânicas do lote de madeira de Eucalyptus.

Utilizou-se a madeira de Eucaliptos, provavelmente E. grandis, adquirida no mercado local secada em estufa e aclimatizada em Viçosa,MG. No ato do recebimento do lote, pôde-se perceber uma grande diferença no peso das tábuas que o constituíam, indicando uma possível variação na densidade da madeira, já que ela havia sido secada em estufa. Assim, todo o lote foi dividido em dois sublotes, um de madeira mais pesada (sublote A) e outro da mais leve (sublote B).

Para determinação das propriedades da madeira, foi feita a caracterização mínima da resistência dos sublotes, utilizando-se para cada uma das propriedades 12 amostras, num total de 120 corpos-de-prova. Para isso, foram retiradas ao acaso 30 tábuas de cada um dos sublotes (A e B).

Para confecção das amostras, levaram-se em conta a forma e dimensões dos corpos-de-prova, bem como a direção das fibras de acordo com a norma NBR 7190/ 97, sendo, para a confecção de alguns tipos de amostra, necessária a solidarização contínua de partes paralelas de uma mesma tábua, utilizando-se adesivo à prova d'água à base de fenol-formaldeído sob pressão, de acordo com as exigências da NBR 7190/1997, para se atingir a espessura necessária à confecção dos tipos amostrais.

R. Árvore, Viçosa-MG, v.28, n.6, p.889-894, 2004 
Determinou-se o teor de umidade do lote através da diferença da massa inicial e massa seca de cada amostra submetida à secagem na temperatura aproximada de $103{ }^{\circ} \mathrm{C}$, como determinado pela NBR 7190/97.

A densidade aparente foi determinada, segundo Vital (1984), pelo método de imersão em água e determinação do volume $(U=12 \%)$ da amostra, pelo empuxo do líquido registrado em balança de precisão.

A caracterização completa das propriedades de resistência da madeira foi feita de acordo com os métodos de ensaio especificados na ABNT (1997), que exige a determinação dos seguintes valores, a serem referidos à condição-padrão de umidade $(\mathrm{U}=12 \%)$ :

- Resistência à compressão paralela às fibras.

- Resistência à compressão perpendicular às fibras.

- Resistência à tração paralela às fibras.

- Resistência ao cisalhamento paralelo às fibras.

- Resistência à flexão.

A caracterização da rigidez da madeira foi medida pelo valor médio do módulo de elasticidade determinado na fase de comportamento elástico-linear. Para isso, respeitaram-se os métodos de ensaio especificados na ABNT (1997).

\section{RESULTADOS E DISCUSSÃO}

O presente trabalho teve o objetivo de separar um lote de madeira de eucalipto comercializada por uma empresa do setor florestal brasileiro em sublotes A e B, pela diferença de peso das peças. A madeira foi previamente caracterizada de acordo com as normas da NBR 7190/97, quando se obtiveram os resultados médios, bem como os coeficientes de variação, das propriedades físico-mecânicas da madeira de Eucalyptus, como apresentado nas Tabelas 1 a 4 .

Como discutido anteriormente, o lote de madeira como adquirido parecia constituir-se de duas populações de tábuas de densidades diferentes. Determinadas as densidades de corpos-de-prova preparados e ensaiados de acordo com a norma NBR 7190/97, pôde-se constatar que a densidade média da madeira do sublote menos denso B) era de $575 \mathrm{~kg} / \mathrm{m}^{3}$, ao passo que a do segundo sublote (A) atingia $880 \mathrm{~kg} / \mathrm{m}^{3}$, um valor $51 \%$ superior. Os dois valores médios são estatisticamente diferentes $(\mathrm{p}<0,01)$ e podem indicar a presença de espécies de madeira diferentes nos lotes de toras que a empresa fornecedora serra.
Pode-se verificar que a madeira de menor densidade atingiu, em todos os resultados, menor resistência mecânica, em comparação com as madeiras de alta densidade, evidenciando a forte relação entre a densidade da madeira e as suas propriedades mecânicas.

Nos testes de cisalhamento, obteve-se nas madeiras de baixa densidade uma resistência média 28,68\% menor em relação à de alta densidade. Na compressão paralela, essa resistência foi de $20,67 \%$ para menos; nos testes de compressão perpendicular foi, em média, $44,61 \%$ menor. Na madeira de baixa densidade, a resistência média à flexão foi $27,32 \%$ inferior. Apesar de a densidade da madeira influenciar sua rigidez, essa relação mostrou ser influenciada, também, por outras propriedades, porquanto os resultados não seguiram a mesma tendência marcante observada nos de resistência.

Nos testes de compressão paralela, o módulo de elasticidade médio, obtido na madeira do sublote B, foi apenas 5,47 \% inferior ao do sublote A. Nos ensaios de flexão, o módulo de elasticidade da madeira do sublote B (baixa densidade) ficou $18,56 \%$ abaixo do módulo da madeira do sublote A (mais densa). Na tração paralela, verificou-se novamente a dependência da resistência em relação à densidade. A resistência das amostras de baixa densidade (sublote B) exibiu um valor médio $19,55 \%$ menor que a resistência média da madeira de alta densidade (sublote A). Já a média do módulo de elasticidade à tração paralela corrigido atingiu 18.793 $\mathrm{MPa}$, com grande variabilidade nos valores individuais da resistência dos corpos-de-prova tanto do sublote B (baixa densidade) quanto do A (alta densidade). A média do módulo de elasticidade à tração paralela do sublote de baixa densidade foi, porém, apenas $9,68 \%$ menor que a do de alta densidade.

Tabela 1 - Valores médios experimentais e corrigidos (teor de $U=12 \%)$ e desvio-padrão das propriedades físicas da madeira de eucalipto

Table 1 - Experimental mean values and corrected $(U=12 \%)$, and standard deviation of Eucalyptus lumber physical properties

\begin{tabular}{lccc}
\hline $\begin{array}{l}\text { Propriedades } \\
\text { Físicas }\end{array}$ & Densidade & Valor Médio & Desvio-Padrão \\
\hline Densidade & A* & $880 \mathrm{~kg} / \mathrm{m}^{3}$ & $66,59 \mathrm{~kg} / \mathrm{m}^{3}$ \\
& B $^{*}$ & $575 \mathrm{~kg} / \mathrm{m}^{3}$ & $71,07 \mathrm{~kg} / \mathrm{m}^{3}$ \\
& Lote & $721 \mathrm{~kg} / \mathrm{m}^{3}$ & $167,74 \mathrm{~kg} / \mathrm{m}^{3}$ \\
Umidade & A $^{*}$ & $12,48 \%$ & $2,29 \%$ \\
& B & $12,47 \%$ & $1,08 \%$ \\
& Lote & $12,475 \%$ & $1,75 \%$ \\
\hline
\end{tabular}

*A (sublote madeira pesada) e B (sublote madeira leve). 
Tabela 2 - Valores médios experimentais e corrigidos para o teor de umidade de $12 \%$ das resistências mecânicas da madeira de eucalipto

Table 2 - Experimental mean values, corrected to $12 \%$ moisture content of Eucalyptus lumber mechanical strength

\begin{tabular}{|c|c|c|c|c|}
\hline$\overline{\text { Propriedades Mecânicas }}$ & $\begin{array}{l}\text { Densidade } \\
(\mathrm{MPa})\end{array}$ & $\begin{array}{c}\text { Valor Médio } \\
(\mathrm{MPa})\end{array}$ & $\begin{array}{c}\text { Valor Médio } \\
\text { Corrigido } \\
\text { (MPa) U }=12 \%\end{array}$ & $\begin{array}{c}\text { Valor } \\
\text { Característico }\end{array}$ \\
\hline \multirow{3}{*}{ Compressão paralela às fibras $\left(\mathrm{f}_{\mathrm{C} 0}\right)$} & $\mathrm{B}$ & $49,5(3,57)$ & $49,5(3,57)$ & 46,5 \\
\hline & A & $62,4(4,31)$ & $62,4(4,30)$ & 61,9 \\
\hline & Lote & $55,9(5,47)$ & $55,5(6,54)$ & 54,2 \\
\hline \multirow[t]{3}{*}{ Compressão perpendicular às fibras $\left(\mathrm{f}_{\mathrm{C} 90}\right)$} & B & $3,6(0,65)$ & $3,6(0,65)$ & 3,1 \\
\hline & A & $6,4(0,86)$ & $6,5(0,81)$ & 5,7 \\
\hline & Lote & $4,9(1,59)$ & $4,9(1,59)$ & 4,4 \\
\hline \multirow[t]{3}{*}{ Cisalhamento $\left(\mathrm{f}_{\mathrm{v}}\right)$} & B & $8,3(0,96)$ & $8,7(0,96)$ & 7,8 \\
\hline & A & $12,1(1,45)$ & $12,2(1,35)$ & 10,6 \\
\hline & Lote & $10,2(0,65)$ & $10,2(0,65)$ & 9,2 \\
\hline \multirow{3}{*}{ Tração paralela às fibras $\left(\mathrm{f}_{\mathrm{t} 0}\right)$} & B & $75,3(23)$ & $75,3(17,5)$ & 56,96 \\
\hline & A & $93,6(28)$ & $93,6(26,5)$ & 73,74 \\
\hline & Lote & $84,5(28)$ & $84,5(23,85)$ & 65,35 \\
\hline \multirow[t]{3}{*}{ Flexão } & $\mathrm{B}$ & $64,7(9,56)$ & $65,7(10,6)$ & 54,7 \\
\hline & A & $88,0(12,60)$ & $90,4(11,5)$ & 76,1 \\
\hline & Lote & $76,35(16,18)$ & $78,0(15,65)$ & 65,4 \\
\hline
\end{tabular}

O valor entre parênteses refere-se ao desvio-padrão e as médias das densidades são $575 \mathrm{~kg} / \mathrm{m}^{3}$ e $880 \mathrm{~kg} / \mathrm{m}^{3}$, e os teores médios de umidade são 12,48 e $12,47 \%$, respectivamente, nas madeiras de baixa (B) e alta densidades (A).

- Os valores característicos da resistência dos sublotes foram estimados pela expressão: $\left[\begin{array}{cc}F w k= & \frac{f\left(1+f 2+\ldots+f(n / 2-1)-f_{n / 2}\right.}{x} \times 1.1 \\ n / 2-1\end{array}\right]$

Tabela 3 - Valores médios experimentais do teor de umidade de $12 \%$ da rigidez da madeira de eucalipto

Table 3 - Experimental mean values, corrected $12 \%$ moisture content of Eucalyptus lumber rigidez.

\begin{tabular}{lccc}
\hline Propriedades Mecânicas & Densidade & $\begin{array}{c}\text { Valor Médio } \\
(\mathrm{MPa})\end{array}$ & $\begin{array}{c}\text { Valor Médio Corrigido } \\
(\mathrm{MPa}) \text { U=12\% }\end{array}$ \\
\hline "E" à compressão paralela às fibras & $\mathrm{B}$ & $18342(4765)$ & $18412(4904)$ \\
& $\mathrm{A}$ & $19933(2667)$ & $19479(2399)$ \\
& Lote & $19137(3863)$ & $18947(3863)$ \\
"E" à tração paralela às fibras & $\mathrm{B}$ & $16154(2079)$ & $17837(2353)$ \\
& $\mathrm{A}$ & $17827(4465)$ & $19749(5019)$ \\
& Lote & $16990(3512)$ & $18793(3956)$ \\
Módulo de elasticidade à flexão & $\mathrm{B}$ & $13772(1732)$ & $13910(1638)$ \\
& $\mathrm{A}$ & $16778(2117)$ & $17080(1181)$ \\
& Lote & $15275(2436)$ & $15596(2019)$ \\
\hline
\end{tabular}

"E"significa o módulo de elasticidade. O valor entre parênteses refere-se ao desvio-padrão, as médias das densidades são 575 kgcm ${ }^{3}$ e $880 \mathrm{~kg} / \mathrm{m}^{3}$ e os teores médios de umidade são 12,48 e $12,47 \%$, respectivamente, nas madeiras de baixa (B) e alta densidades (A).

Tabela 4 - Valores médios experimentais e corrigidos para o teor de umidade de $12 \%$ das propriedades mecânicas da madeira de Eucalyptus grandis e do lote de madeira de eucalipto e respectivos sublotes

Table 4 - Experimental mean values, corrected to $12 \%$ moisture content of Eucalyptus grandis lumber mechanical properties and the lot of Eucalyptus wood and their respective sublots

\begin{tabular}{|c|c|c|c|c|c|c|c|}
\hline $\begin{array}{l}\begin{array}{l}\text { Nome Comum } \\
\text { (dicotiledôneas) }\end{array} \\
\end{array}$ & Nome Científico & $\begin{array}{c}\rho_{\mathrm{ap}}(12 \%)^{1} \\
\mathrm{~kg} / \mathrm{m} 3)\end{array}$ & $\begin{array}{l}\mathrm{F}_{\text {co }}{ }^{2} \\
\text { MPA }\end{array}$ & $\begin{array}{l}\mathrm{F}_{\text {to }}^{3} \\
\text { MPA }\end{array}$ & $\begin{array}{l}F_{v}^{4} \\
\text { MPA }\end{array}$ & $\begin{array}{l}E_{\text {co }} 5 \\
\text { MPA }\end{array}$ & $\mathrm{n}^{6}$ \\
\hline E. grandis* & Eucalyptus grandis & 640 & 40,3 & 70,2 & 7,0 & 12813 & 103 \\
\hline Sublote B (baixa densidade) & - & 575 & 49,5 & 75,3 & 8,7 & 18412 & 12 \\
\hline Sublote A (alta densidade) & - & 880 & 62,4 & 93,6 & 12,2 & 19479 & 12 \\
\hline Lote (sublote $A+B)$ & & 721 & 55,5 & 84,5 & 10,2 & 18947 & 24 \\
\hline
\end{tabular}

*Fonte: Anexo E da NBR 7190/1997.

${ }^{1} \rho(12 \%)$ é a massa específica aparente a $12 \%$ de umidade. ${ }^{2} \mathrm{~F}$ é a resitência à comprenssâo paralela às fibras. ${ }^{3} \mathrm{~F}$ é a resitência à tração paralela às fibras. ${ }^{4} \mathrm{~F}$ é a resitência ao cisalhamento. ${ }^{5} \mathrm{E}$ é $\mathrm{o}$ o módulo de elasticidade longitudinal obtido no ensaio de compressão paralela às fibras. ${ }^{6} \mathrm{n}$ é o número de corpos-de-prova ensaiados. 


\section{CONCLUSÕES}

- O estudo da caracterização da madeira de eucalipto utilizando a norma NBR/7190 (1997) indicou que a densidade influencia intensamente a resistência mecânica da madeira, e através da medição dessa propriedade foi possível inferir a existência de pelo menos duas populações de tábuas no lote analisado.

- Ao final da análise foi concluído que os valores médios das propriedades de resistências dentro do sublote da madeira de baixa densidade (sublote B) nos testes realizados ficaram próximos aos da madeira de Eucalyptus grandis apresentados no Anexo E1 da ABNT (1997) e que é apresentada na Tabela 4 deste artigo. Já os valores médios obtidos no sublote da madeira de alta densidade (sublote A) e de todo o lote apresentaram-se muito acima desses. Portanto, foi possível inferir que o sublote A se tratava de uma espécie diferente da existente no sublote $\mathrm{B}$ e que esta última provavelmente se referia à espécie E. grandis.

- Quanto ao uso de lotes de madeira com grandes variações de densidade, viu-se a necessidade de uma avaliação preliminar para agregação de sublotes com valores de densidades mais homogêneos, a fim de que o uso desse sublote de madeira seja compatível com as resistências de utilização correspondentes.

\section{REFERÊNCIAS BIBLIOGRÁFICAS}

\section{ASSOCIAÇÃO BRASILEIRA DE NORMAS TÉCNICASS (ABNT). Projetos de estruturas de madeira - NBR 7190. Rio de Janeiro: 1997. 107p.}

EVANS, J.L.W.; SENFT, J. F.; GREEN, D. W. Juvenile wood effect in red alder: analysis of physical and mechanical data to delineate juvenile and mature wood zones. Forest Products Journal, v.50, n.7/8, p.75-87, 2000.
GONÇALEZ, J.C. Caracterisation tecnologique de quatre espéces peu connues de la Fôret Amazonienne: Anatomie, Chimie, Couleur, Propriétes physiques e méchaniques. 1993. 444f. (Thése de Doctorat). École Nationale du Génie Rural des Eaux et des Fôrets (ENGREF), Nancy, 1993.

LATORRACA, J.V.; ALBUQUERQUE, C.E.C, . Efeito de rápido crescimento sobre as propriedades da madeira. Revista Floresta e Ambiente, v.7, n.1, p.279-291, 2000.

NORMAN, D. Manuel d'identification des bois comerciaux. CTFT, 1972, 193p.

PANSHIN, A.J.; DE ZEEUW, C. Textbook of wood technology. 4 ed. New York: 1980. 722p.

EUCALIPTO, a madeira do futuro. Revista da Madeira, setembro, 2001. 114p. (Edição especial).

EUCALIPTO, pesquisa amplia usos. Revista da Madeira, agosto, 2003. 138p. (Edição especial).

SHIMOYAMA, V.R. Variações da densidade básica e características anatômicas e químicas da madeira em Eucalyptus sp. 1990. 93p. Dissertação (Mestrado em Ciência Florestal). Escola Superior de Agricultura Luiz de Queiroz, Piracicaba, 1990.

TOMAZELLO FILHO, M. Variação radial da densidade básica e da estrutura anatômica da madeira do $E$. saligna e $E$. grandis. IPEF, n.29, p.37-45, 1985a.

VITAL, B. R. Métodos de determinação da densidade da madeira. Viçosa, MG: Sociedade de investigações florestais, 1984. 21p. (Boletim Técnico, 1). 